\title{
Conduction Recovery with Wenckebach Periodicity at the Mitral Isthmus
}

\author{
Takashi Nakashima ${ }^{1}$, Thomas Pambrun ${ }^{1}$, Gabriela Pintican ${ }^{1}$, Takamitsu Takagi ${ }^{1}$, Pierre \\ Jais $^{1}$, and Nicholas Derval ${ }^{1}$ \\ ${ }^{1}$ University of Bordeaux
}

November 10, 2020

\begin{abstract}
A Wenckebach periodicity at the mitral isthmus lesion has rarely been reported. We described a case presenting conduction recovery with Wenckebach periodicity at the mitral isthmus lesion after achieving the posterior mitral isthmus block. These findings demonstrate an early reconnection of mitral isthmus lesion, and an arrhythmogenic substrate that can lead to development of reentrant tachycardia, that is, peri-mitral atrial flutter.
\end{abstract}

\section{Conduction Recovery with Wenckebach Periodicity at the Mitral Isthmus}

\section{Short title: Wenckebach conduction of mitral isthmus}

Takashi Nakashima, MD; Thomas Pambrun, MD; Gabriela Pintican, MD; Takamitsu Takagi, MD; Pierre Jaïs, MD; Nicolas Derval, MD

LIRYC, University of Bordeaux, CHU de Bordeaux, Bordeaux France

Correspondence to Takashi Nakashima, MD

Service de Rythmologie, Hôpital Cardiologique du Haut-Lévêque (Centre Hospitalier Universtaire de Bordeaux)

Avenue de Magellan, 33604, Bordeaux-Pessac, France

Tel: +33557656863 19, Fax: +3355765689620

Email; takashin727jw@yahoo.co.jp

ORCID: 0000-0002-8538-9259

Conflicts of interest : none

Funding : none

Key words

Atrial fibrillation; mitral isthmus; Wenckebach conduction; ablation; conduction block

\section{Abstract}

A Wenckebach periodicity at the mitral isthmus lesion has rarely been reported.

We described a case presenting conduction recovery with Wenckebach periodicity at the mitral isthmus lesion after achieving the posterior mitral isthmus block. These findings demonstrate an early reconnection of mitral 
isthmus lesion, and an arrhythmogenic substrate that can lead to development of reentrant tachycardia, that is, peri-mitral atrial flutter.

\section{Case presentation}

A 66-year-old man was referred for a third procedure for recurrent atrial fibrillation (AF) after two previous failed pulmonary vein isolations (PVIs). During the procedure, the right pulmonary vein was reconnected and re-isolated. The linear ablations targeting the left atrial roof, posterior mitral isthmus (MI), and cavotricuspid isthmus were also performed. After radiofrequency catheter ablation (RFCA) from the coronary sinus (CS), the atrial electrograms (EGMs) recorded in the CS changed to a proximal-to-distal activation sequence, and MI blocked was confirmed by the differential pacing maneuver (Figures 1A and 1B). The MI was then reconnected during the observation period (Figure 1C). Electrograms (EGMs) recorded in the distal part of the CS demonstrated double components during pacing from the left atrial appendage at a pacing cycle length (CL) of $600 \mathrm{~ms}$. The first one was a low amplitude and frequency signal demonstrating a distal-to-proximal activation sequence, indicating the far-field left atrium (LA) EGMs. The second one was a high amplitude and frequency signal demonstrating both middle-to-distal and middle-to-proximal activation sequences, indicating the near-field CS EGMs. The interval between the stimulus and the first component recorded in the CS 1-2 to CS 5-6 gradually prolonged and shortened again, suggesting a Wenckebach periodicity. This phenomenon was repeatedly observed. Consolidation RFCA was performed to the endocardial MI lesion, resulting in MI block again.

The rate-dependent conduction block of the MI line has been reported as a potential pitfall in the assessment of MI block. ${ }^{1}$ However, Wenckebach periodicity at the MI lesion has rarely been reported. An incomplete MI block increases the risk of developing peri-mitral atrial flutter (PM-AFL), which is the most frequent post-AF ablation macro-reentrant tachycardia. ${ }^{2}$

Both slow conduction and unidirectional block contribute to initiate and maintain the reentrant tachycardia. ${ }^{3}$ It is noteworthy in the present case that it described a Wenckebach periodicity at the endocardial posterior MI lesion presenting with gradual conduction delay, and subsequent conduction block and conduction recovery. Because these findings demonstrate an early reconnection of MI lesion, and an arrhythmogenic substrate that can lead to development of reentrant tachycardia, that is, PM-AFL.

\section{References}

1. Chen S, Lu X, Wei Y, Liu S. Rate-dependent conduction block: A pitfall in the assessment of mitral isthmus conduction block. J Cardiovasc Electrophysiol . 2019;30:2558-2560.

2. Sawhney N, Anand K, Robertson CE, Wurdeman T, Anousheh R, Feld GK. Recovery of mitral isthmus conduction leads to the development of macro-reentrant tachycardia after left atrial linear ablation for atrial fibrillation. Circ Arrhythm Electrophysiol . 2011;4:832-7.

3. Kléber AG, Rudy Y. Basic mechanisms of cardiac impulse propagation and associated arrhythmias. Physiol Rev . 2004;84:431-88.

\section{Figure legends}

\section{Figure 1.}

Electrograms (EGMs) during left atrial appendage (LAA) pacing at a pacing cycle length of 600 ms demonstrating posterior mitral isthmus (MI) block and its reconnection with an endocardial conduction gap.

(Left panel) Before achieving MI block. (Middle panel) After achieving MI block. Atrial EGMs recorded in the coronary sinus (CS) demonstrate a proximal-to-distal activation sequence. MAP is located at the endocardial MI lesion. The interval between the stimulus and EGM recorded on the MAP is $190 \mathrm{~ms}$. (Right panel) Atrial EGMs recorded in the CS showing the reconnection of MI lesion. EGMs in the CS showing double components: far-field left atrium (LA) EGMs with low amplitude and frequency (arrows) precede the 
near-field CS EGMs with high amplitude and frequency (arrowheads). LAA pacing demonstrates a distalto-proximal activation sequence of the LA (arrows) followed by middle-to-distal and -proximal activation sequences of the CS (arrowheads). MI, mitral isthmus; S, stimulus.

\section{Figure 2.}

EGMs during LAA pacing at a pacing cycle length of $600 \mathrm{~ms}$ demonstrating a Wenckebach periodicity at the endocardial MI lesion. Note that the intervals between the stimulus and far-field LA EGMs recorded in the CS 1-2 to CS 5-6 gradually prolong and shorten again (numbers), indicating a Wenckebach periodicity at the posterior MI lesion.

\section{Figures}

\section{Figure 1}


\section{Figure 2}

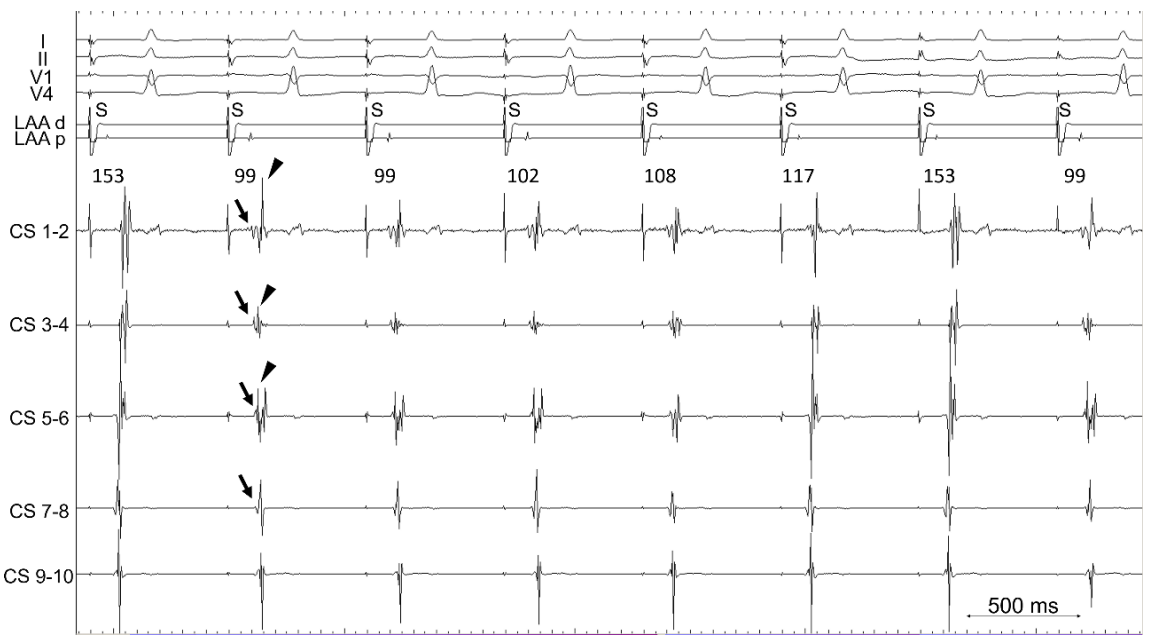

